\title{
Impact of Limits in Pathways Between Sinoatrial Node and Atrium on Heart Rhythm by Timed Automata Model
}

\author{
D Makowiec ${ }^{1}$, ZR Struzik ${ }^{2,3}$ \\ ${ }^{1}$ Institute of Theoretical Physics and Astrophysics, University of Gdańsk, Poland \\ ${ }^{2}$ RIKEN Brain Science Institute, Japan \\ ${ }^{3}$ Graduate School of Education, University of Tokyo, Japan
}

\begin{abstract}
There are evidences that the human right atrium and sinoatrial node (SAN) are functionally separated except at discrete SAN-atrial electrical junctions. We hypothesize that such anatomy could be a source of re-entry around the SAN.

A model was developed which reconstructs the human right atrium anatomy. The activity of a myocyte was simulated by a timed automaton with continuous and discrete transitions reproducing the known stages of the action potential of the cellular membrane. A stochastic $2 D$ network of timed automata was designed to model the grid of the right atrium. Discrete modeling allowed us to specify directly the rate of SAN to atrium pathways. Also, the influence of (1) atrial tissue fibrosis, via probability for transversal intercellular network connections, and of (2) impairment of individual cells via probability of a cell to excite, were controlled.

The simulations provided a critical relationship between atrial anatomy and the rhythm of heart excitations. It occurred that at probability of 1/8 of SAN-atrium pathways (randomly chosen), the occurrence of the normal rhythm attained the highest probability - close to 1, in large intervals for the density of transversal intercellular connections, and for the levels of cellular impairment.
\end{abstract}

\section{Introduction}

The sinoatrial node (SAN) is the primary pacemaker of the heart, what means that the SAN is responsible for initiating cardiac impulse. There are two crucial properties that make the SAN special. The automaticity of each of the SAN cell and stochastic, and rather free, structure of intercellular connections when compared to the structure of atrial cell connections. The SAN is located in the upper part of the right atrium close to the opening of of the superior vena cava, on the right atrium (RA). There are contradictory hypothesis of how the SAN is electrically con- nected to the atria :

- the SAN is electrically insulated from the surrounding atria by a structural border of fibrosis, fat layers, and myocyte discontinuity, and the functional and structural connection between the SAN and atria is limited to discrete SAN exit pathways [1]

- the SAN and atrial cells are extensively connected by diffuse digitations of the SAN border with the atrial myocardium, and no discrete pathways exists [2].

In simulations we test the effect of limits in paths connecting the SAN cells with the RA cells.

Podziemski and Żebrowski in [3] proposed a simplified, yet fully physiologically and numerically justified, model of the atria. It operates on the 2D grid of cells divided into dedicated regions of the SAN, atroventricular node (AVN) and regions of normal atrial conductive tissue in between. The ion-channel cardiac cell activity was driven by the differential equations. In particular, popular sets of equations of Fenton-Karma and FitzHugh-Nagumo were used. However, there is a modern computational technique, called hybrid automata (HA), allowing to preserve all properties of the dynamical system by separating the evolution into continuous parts and transitions between them [4]. Intuitively, short-lived, transient behaviors are represented as discrete transitions [5].

In [6] we proposed the model of the right atrium which takes advantages of HA technique. In the following we consider the time automata (a type of hybrid automata) approach allowing to model directly the pathways between the SAN and RA, and allowing to study the impact of the density of these paths on establishing the normal heart rhythm. Also, we will present results of simulations testing the influence of tissue fibrosis and impairment of individual cells. The simulations provided a critical relationship between atrial anatomy and the rhythm of heart excitations. It occurred that at probability of $1 / 8$ of SANatrium pathways (randomly chosen), the occurrence of the normal rhythm attained the highest probability - close to 1 , in large intervals for the density of transversal intercellu- 


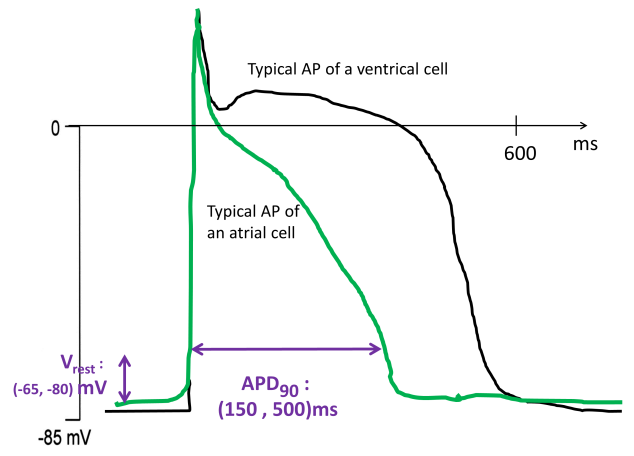

Figure 1. Membrane AP for typical myocytes of atrium (green) and ventricle (black). Given values of uncertainty in the shape of AP follows [7].

lar connections, and for the levels of cellular impairment.

\section{Methods}

\subsection{Timed automata model of an atrial cell}

The method of HA relies on mapping the time development of a system into automata states and transitions between them [4]. Sets of differential equations drive the evolution in the states. In case of cardiac tissue these equation reproduce the shape of the myocyte AP, see Figure 1.

It is easy to see that in reconstruction of the triangular shape of the AP of the atrial myocyte, the three states are enough. The first state describes a cell staying in the resting potential $V_{R}$. The second state is the potential upstroke increase which occurs in response to some external stimulation $V_{S}$. The third state corresponds to the decay of the membrane potential to the resting value.

It occurs that independently of the sets of differential equations assigned to the particular state, the real variables behave regularly $[8,9]$. Namely, the state evolution depends on time in a linear way. One can say that the state properties, here the potential value, depend proportionally to the time spent by a cell in the state. Consequently, there is no reason to solve computationally demanding differential equations. This observation leads to the notion of timed automata $[10,11]$.

Formally, for a finite set of environment events $\Sigma$, a timed automaton is defined as a tuple of [9]: $\mathcal{A}=$ $\{G, \mathcal{C}, \operatorname{init}(), \operatorname{inv}()$, jump (), event ()$\}$. The elements of the tuple $\mathcal{A}$ are described as follows:

(i) $G=(V, E)$ is a directed graph with a set of vertices $V$, called states, and a set of edges $E$, called transitions;

(ii) $\mathcal{C}=\left\{x_{1}, \ldots x_{n}\right\}$ is a set of real-valued variables, called clocks, which in each time step advance their value by 1 or reset it to 0 ; (iii) set of functions labeling graph vertices: init $\subset \Sigma$, inv : $V \rightarrow C l o c k C o n s t r i a n t s$, and graph edges: event : $V, E \rightarrow \Sigma$, jump.Guard $: E \rightarrow$ ClockConstriants and jump.Action : $E \rightarrow$ ClockReset.

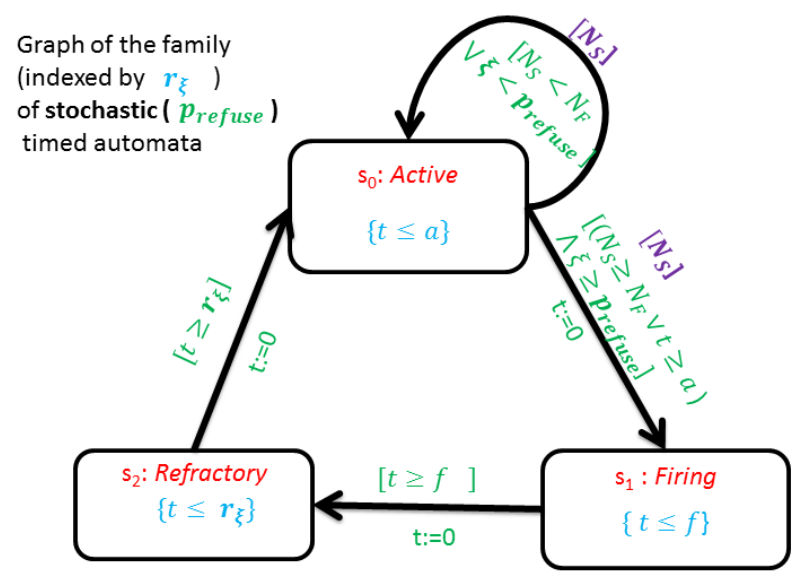

Figure 2. Stochastic TA which follows the Luo-Rudy dynamics. The extra condition for cellular excitation is introduced to mimic cellular impairment. The excitation in the presence of strong external enough external stimulation $\left[N_{S} \geq N_{F}\right]$ is performed with probability $p_{\text {refuse }}$.

The timed automaton, representing the myocyte dynamics, defined on three states, can be described by one clock variable $t$, and by four edges, see Figure 2. The jumps are driven by the three model constants, ClockConstraints, namely $a, f, r_{\xi}$ which mean $a$ - the length of $s_{0}$ state, $f$ - the length of $s_{1}$ state, and $r_{\xi}$ the length of APD. In our proposition $r_{\xi}$ is a random variable, assigned to each automaton individually. The only external event, the stimulation by $N_{S}$ neighboring cells being in $s_{1}$ state, governs the transition between states $s_{0} \rightarrow s_{1}$. Our automaton mimics the cellular intrinsic cycle with period $T=a+f+r_{\xi}$. In case $a=\infty$ the AP cycle is evoked by the external stimulation $N_{S} \geq N_{T}$. The random values of $r_{\xi}$ mimic heterogeneity between atrial cells.

Additionally, to reproduce a possible impairment/fatigue of a cell, stochasticity into the TA transition $s) \rightarrow s_{1}$ was introduced, see the edge label in Figure 2. The transition is constrained by the probability $p_{\text {refuse }}$.

\subsection{The network used to model the right atrium.}

We assume that TA cells are arranged in nodes of a square lattice. The size of the lattice $L$ should match the SAN cell period, namely $T_{\mathrm{SAN}}=f_{\mathrm{SAN}}+r_{\mathrm{SAN}}+a_{\mathrm{SAN}}$, to guarantee that one wave front is expected to propagate over the lattice in any time step. 


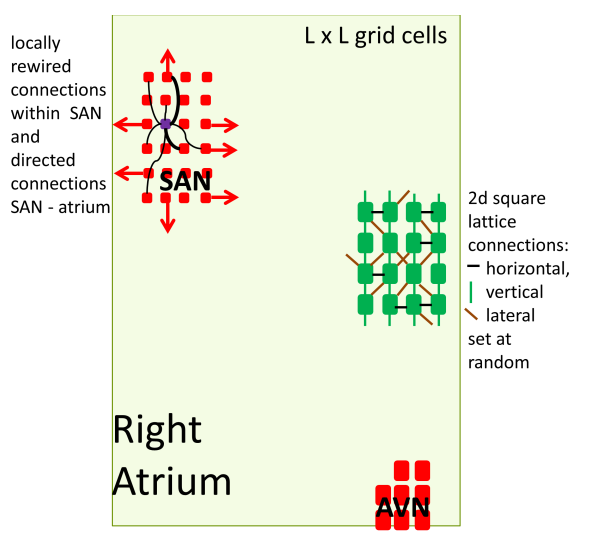

Figure 3. The right atrium structure used in simulations. The lattice size is $L=120$. The SAN consists of $5 \times 15$ cells down from $(10,10)$. The AVN is built from eight cells up from $(100,120)$. The AVN is excited if two of eight AVN cells are simultaneously excited. The intrinsic dynamics of the atrial cell is driven by time constrains $f=1, r_{0}=50, a=1000$ and of the SAN cell: $f_{\mathrm{SAN}}=10, r_{0, \mathrm{SAN}}=50, a_{\mathrm{SAN}}=80$. These values are chosen as best fitting to the physiological facts.

Each cell can interact with its neighbors from the Moore neighborhood: two vertical, two horizontal and four lateral ones, see Figure 3. We assume that all atrial cells are connected vertically to reproduce the known facts about atrial architecture. Density of connections in other directions: horizontal and lateral are the model parameters. In case of cells in the SAN, there is no direction in favor.

The bordering cells of the SAN are in general isolated from the atrial cells. Some of them, selected with probability $p_{\mathrm{SAN} \text { exits }}$ have a connection directed from the SAN cell to the neighboring atrial cell.

\section{Results and discussion}

In a series of plots (A), (B) and (C) we show typical wave fronts observed in simulations. Each row displays the same state but at distinct moments of time. Assuming that each AP propagates unperturbed in the remaining part of the heart, the time steps between subsequent AVN excitations are considered as intervals of heart beats. Accordingly, we call the state shown in (A) the normal rhythm, in (B) SAN re-entry, and in (C) other arrhythmia.

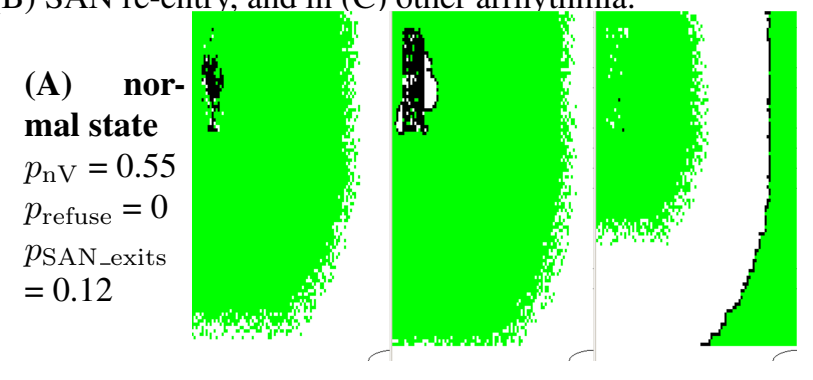

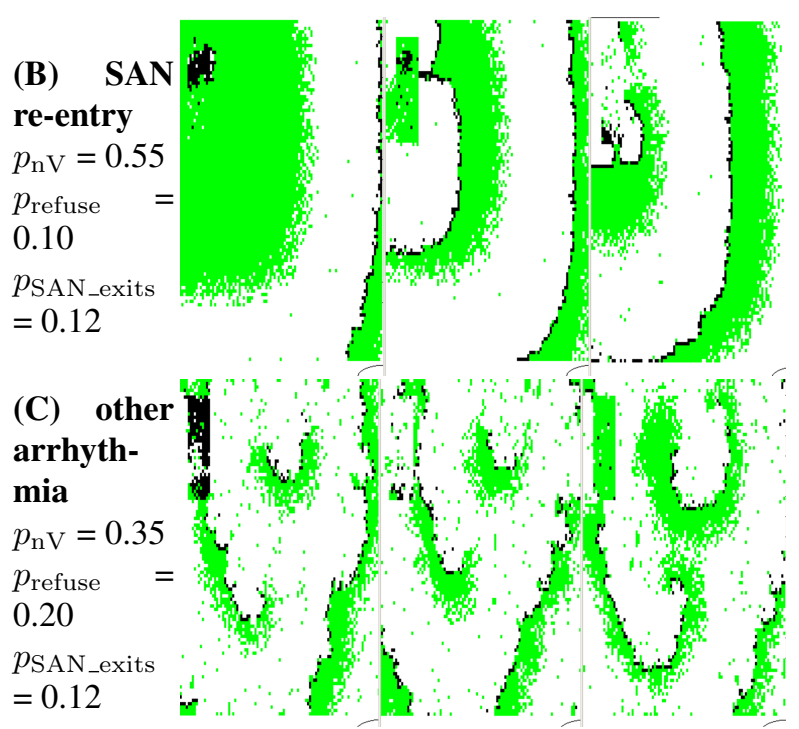

The limit states were observed for many densities of not ventricular connections, $p_{n V}=p_{\text {horizontal }}=p_{\text {lateral }} \in$ $(0.05,0.90)$, for different levels of cellular impairment $p_{\text {refuse }} \in[0.0,0.5]$, and at different ratio $p_{\text {SAN_exit }}$ of SAN to atrium connections. The ADP values $r_{\xi}$ were uniformly distributed in the interval $[30,40]$.

Each parameter setting was simulated a hundred times. After an initial one thousand steps allowed for the system stabilization, the state classification was performed according to the number of wavefronts reaching the AVN during next 3000 steps. The ratios of normal rhythms observed for different parameter settings are shown in Figure 4.

From the plots of Figure 4 one can see that probability for the stabilization at the normal rhythm depends on both the density of non vertical connections $p_{n V}$, and probability to refuse of an individual cell $p_{\text {refuse. It is noticeable that }}$ the level of this probability is related to the number of paths from the SAN to atrium $p_{\mathrm{SAN} \text { _exit }}$. It occurs that the most stable system, in a sense the probability to obtain the normal rhythm, is attained when $p_{\text {SAN_exit }} \in(0.25,0.06)$. It occurs that the region with the highest probability to obtain the normal rhythm switches critically to 0 with decrease of non vertical connections and increase of $p_{\text {refuse. }}$ The line of the transition can be approximated as follows

$$
p_{\text {refuse }}\left(p_{n V}\right)=p_{n V}\left(1-0.5 p_{n V}\right)
$$

what indicates that cellular impairment has a weaker effect on the impulse propagation than the lost of transversal connections. This critical dependence is present also for other values of $p_{\text {SAN_exit }}$. However, with departure from $p_{\text {SAN_exit }}=0.125$ there is observed an increase presence of the SAN re-entry states.

\section{Summary}

In case of the AP of a myocyte the HA approach leads to numerically indistinguishable results from solutions ob- 

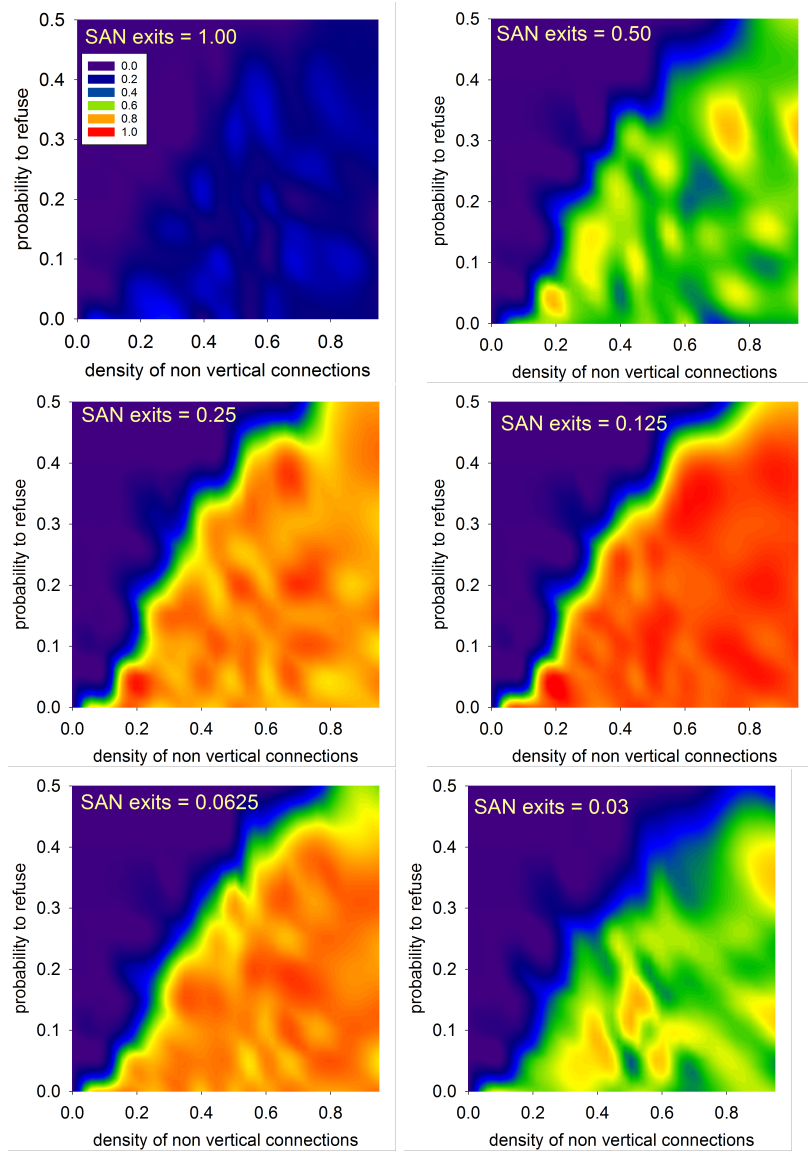

Figure 4. Ratio of normal rhythms observed in stationary state when the simulation is run with different density of non vertical connections and different probability $p_{\text {refuse }}$ to refuse for stimulation, for different ratio of connections between the SAN and atrium.

tained by the continuous methods. But efficiency of such simulations is ten times better than in case of the continuous models [9]. Additionally, the HA approach allows to manipulate with heterogeneity of cellular dynamics.

Our simulations have provided a critical relationship between atrial anatomy and the rhythm of heart excitations. It occurred that at probability of about $1 / 8$ of possible SANatrium pathways (randomly chosen), the occurrence of the normal rhythm attained the highest probability — close to 1. This property is valid in large intervals for the density of transversal intercellular connections, and for the levels of cellular impairment. The same critical relationship has been obtained with the SAN size doubled, namely, when the SAN consisted with $10 \times 30$ cells.

\section{References}

[1] Efimov IR, Fedorov VV, Joung B, Lin SF. Mapping cardiac pacemaker circuits: Methodological puzzles of the sinoatrial node optical mapping. Circulation Research 2010; 3(106):255-271.

[2] Sánchez-Quintana D, Cabrera JA, Farré J, Climent V, Anderson RH, Ho SY. Sinus node revisited in the era of electroanatomical mapping and catheter ablation. Heart 2005; 91(2):189-194. ISSN 1355-6037.

[3] Podziemski P, Żebrowski JJ. A simple model of the right atrium of the human heart with the sinoatrial and atrioventricular nodes included. Journal of Clinical Monitoring and Computing Aug 2013;27(4):481-498. ISSN 1573-2614.

[4] Sloth C, Wisniewski R. Complete abstractions of dynamical systems by timed automata. Nonlinear Analysis Hybrid Systems 2013;7(1):80 - 100. ISSN 1751-570X. IFAC World Congress 2011.

[5] Chen T, Diciolla M, Kwiatkowska M, Mereacre A. A simulink hybrid heart model for quantitative verification of cardiac pacemakers. In Proceedings of the 16th International Conference on Hybrid Systems: Computation and Control, HSCC '13. New York, NY, USA: ACM. ISBN 978-1-4503-1567-8, 2013; 131-136.

[6] Makowiec D, Wdowczyk J, Struzik ZR. Heart rhythm insights into structural remodeling in atrial tissue: timed automata approach. Frontiers in Physiology 2018; under consideration.

[7] Fatkin D, Otway R, Vandenberg JI. Genes and atrial fibrillation. Circulation 2007;116(7):782-792. ISSN 0009-7322.

[8] Ye P, Entcheva E, Grosu R, Smolka SA. Efficient modeling of excitable cells using hybrid automata. In Proceedings of Computational Methods in System Biology. 2005;216-227.

[9] Ye P, Entcheva E, Smolka S, Grosu R. Modelling excitable cells using cycle-linear hybrid automata. IET Systems Biology January 2008;2:24-32(8). ISSN 1751-8849.

[10] Bartocci E, Corradini F, Merelli E, Tesei L. Detecting synchronisation of biological oscillators by model checking. Theoretical Computer Science 2010;411(20):1999 - 2018. ISSN 0304-3975. Hybrid Automata and Oscillatory Behaviour in Biological Systems.

[11] Wisniewski R, Sloth C. Abstractions of dynamical systems by timed automata. Modeling Identification and Control 02 2011;32:79-90.

Address for correspondence:

Danuta Makowiec

Institute of Theoretical Physics and Astrophysics of Gdansk University, 80-308 Gdańsk, ul. Wita Stwosza 57, Poland

e-mail: danuta.makowiec@ug.edu.pl 